\title{
X-ray topographic study of growth defects of trans-stilbene crystals grown from
} solutions.

\author{
Helmut Klapper ${ }^{1, *}$, Natalia Zaitseva ${ }^{2}$, Leslie Carman ${ }^{2}$ \\ ${ }^{1}$ Institut für Kristallographie, RWTH Aachen University, D-52066 Aachen, Germany \\ ${ }^{2}$ Lawrence Livermore National Laboratory, 7000 East Avenue, Livermore, CA94551, USA
}

\begin{abstract}
Single crystals of trans-stilbene, $\mathrm{C}_{14} \mathrm{H}_{12}$, with properties suitable for high-energy neutron detection were grown from solution in anisole and toluene by the temperature reduction method with growth rates up to $6 \mathrm{~mm} /$ day. From these crystals, slices of appropriate orientation and thickness of $2-4 \mathrm{~mm}$ were cut and studied by X-ray diffraction topography applying the Lang method using $\mathrm{CuK} \alpha$ radiation. The topographs exhibit growth defects such as liquid inclusions, dislocations, striations, and faulty growth-sector boundaries. These defects occur in the same typical arrangements and geometries as is observed in all kinds of crystals grown on habit faces from solution. Besides growth dislocations originating from inclusions and propagating with the growth front, many plastic glide dislocations in the shape of loops or half-loops emitted from inclusions by stress relaxation are observed. The glide system underlying this plasticity is discussed.
\end{abstract}

Keywords: A1. X-ray topography, A1. Dislocation, A2. Growth from solutions, B1. Aromatic crystal, B1. Trans-stilbene, B2. Organic scintillator

\footnotetext{
* Corresponding author.

E-mail address: klapper@xtal.rwth-aachen.de (Helmut Klapper)
} 


\section{Introduction}

Organic molecular crystals, in particular those of fluorescent aromatic compounds, may exhibit scintillation properties which can be used for detection of different types of radiation [1]. The high content of hydrogen in their composition makes aromatic materials particularly useful for direct detection of high-energy neutrons discriminated from strong gamma radiation background $[2,3]$. The mechanism that involves elastic scattering of neutrons on hydrogen nuclei with production of scintillation pulses has been tested with a variety of aromatic crystals $[4,5]$, among which single crystal trans-stilbene was found to be the best material due to its remarkable pulse shape discrimination (PSD) properties [6] not challenged so far by any other types of organic scintillators. Relatively small crystals (up to $10 \mathrm{~cm}$ ) of transstilbene have been traditionally grown from melt by the Bridgman technique [7], but due to the high thermal expansion and low mechanical strength of these crystals, the thermal gradients and the clamping contact with the ampoule wall involved in this method lead to formation of a defective mosaic structure, with varying degrees of block disorder [8].

Recently Carman et al. [9] and Zaitseva et al. [10] showed that high-optical quality crystals of stilbene can be grown to a $10-\mathrm{cm}$ scale from solution. Some current and future applications may require larger size and quantity of crystals, production of which can be a complicated task without detailed knowledge of the growth mechanisms and structure, to avoid formation of defects harmful for the final application. Here we report results of X-ray topographic investigations of trans-stilbene that represents the first pure aromatic crystal grown on a commercial scale [11] from organic solutions. Although the present study is concentrated only on stilbene, the results may be of general interest for exploration of a practically endless 
variety of organic crystals that can be grown by similar methods for different applications [12-14].

\section{Crystallographic data and physical properties}

Trans-stilbene $\left(\mathrm{C}_{14} \mathrm{H}_{12}\right)$ crystallizes in the monoclinic centrosymmetric point group $2 / m$. Early descriptions of the symmetry and morphology of crystals precipitated from various organic solvents have been reported by Groth in 1919 [15]. The first structure determination was done in 1937 by Robertson \& Woodward [16], followed in 1974 and 1975 by refinements by Finder et al. [17], Bernstein [18] and Hoekstra et al. [19], who used different settings of the monoclinic co-ordinate axes. In the following we use the settings of Finder et al. $(a=$ $15.710 \AA \AA$, $b=5.723 \AA \AA$, $c=12.381 \AA$, $\beta=111.89^{\circ}$, space group $P 2{ }_{1} / c$ ). The molecular packing of trans-stilbene is shown in Fig. 1a. The typical morphology of crystals slowly grown from solution in toluene or anisole is given in Fig. 1b. Trans-stilbene melts at $\sim 124^{\circ} \mathrm{C}$. It exhibits a high anisotropy of thermal expansion, which varies by a factor of about 2 at room temperature [20, 21]. The anisotropy of the elastic constants, however, is moderate [21].
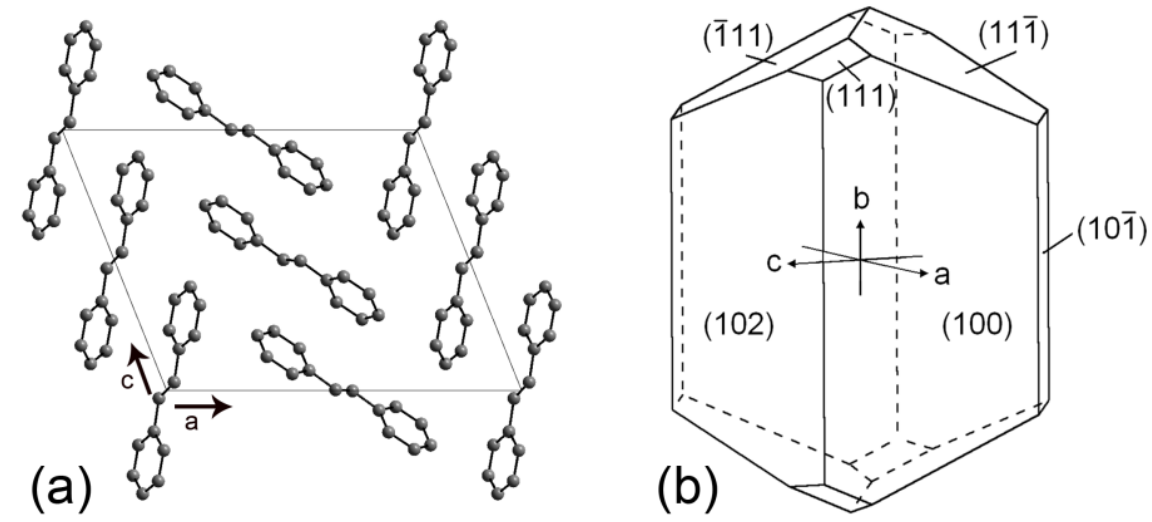

Figure 1: (a) Packing of trans-stilbene molecules $\mathrm{C}_{6} \mathrm{H}_{5}-\mathrm{CH}=\mathrm{CH}-\mathrm{C}_{6} \mathrm{H}_{5}$ (only carbons are shown), projected along the monoclinic axis. (b): Typical shape of trans-stilbene crystals grown from solutions in toluene or anisole with faces $\{100\},\{102\},\{11 \overline{1}\},\{111\}$ and $\{10 \overline{\mathbf{1}}\}$ (indices refer to the co-ordinate system of Finder et. al. [17]). With a small acceleration of growth rate, the facets $\{111\}$ and $\{10 \overline{1}\}$ usually grow out and vanish from the morphology. 


\section{Crystal growth}

Crystals for investigations were grown by a method used previously for growth of potassium dihydrogen phosphate $\mathrm{KH}_{2} \mathrm{PO}_{4}(\mathrm{KDP})$ crystals [22-24], with the basic equipment modified to withstand the reactivity of organic solvents [12]. The details of these modifications, as well as major procedures and requirements of material purity were described in a recent publication [9]. By analogy with the rapid growth of KDP [22], in the first experiments a small "point" seed glued in the centre of the platform crystal-holder with vertical position of the monoclinic b-axis was used. The platform was introduced into the solution heated above the saturation temperature that then was decreased to start growth of fully faceted crystals. It was very soon established that to obtain such crystals without visible solution inclusions was possible only at extremely slow growth rates below $\sim 1 \mathrm{~mm} /$ day (Figure 2a). Any attempts to increase growth rate further resulted in massive defect formation on the $\{11 \overline{1}\}$ faces (Figure 1b), making

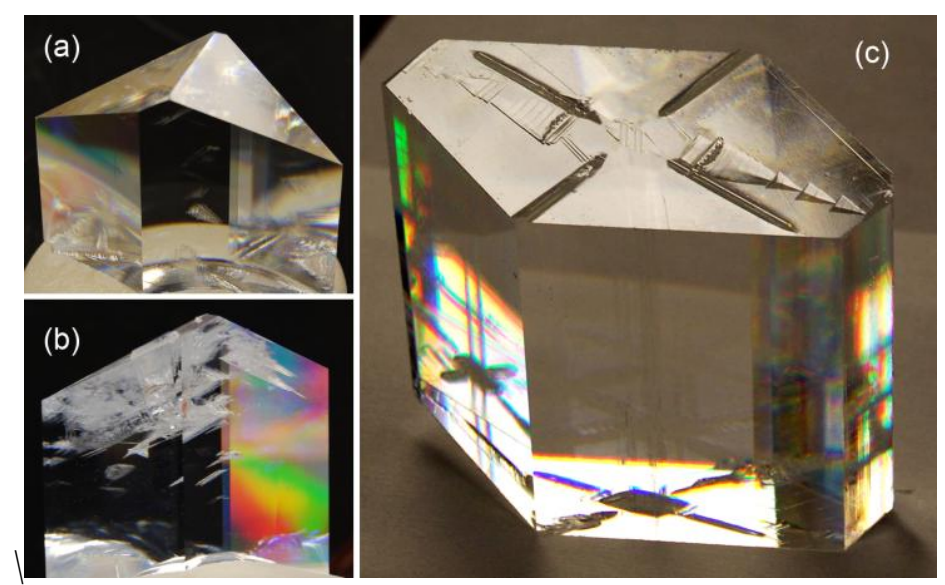

Figure 2: trans-Stilbene single crystals grown from anisole solutions (monoclinic axis vertical): (a) slowly ( $<1 \mathrm{~mm} /$ day) grown with only few solution inclusions formed on the $\{11 \overline{\mathbf{1}}\}$ faces; (b) grown with higher growth rate ( $>1 \mathrm{~mm} /$ day) showing massive formation 
of liquid inclusions on $\{11 \overline{\mathbf{1}}\}$ faces; (c) $10 \mathrm{~cm}$ tall crystal grown without $\{11 \overline{\mathbf{1}}\}$ faces, growth of which was restricted by the platform design [9].

grown crystals unsuitable for any optical tests or scintillation applications. The problem was resolved by the development of a special platform design described in a previous report [9]. In this design, a thin rod-shaped seed cut along the monoclinic axis [010] with the length close to the desired height of the final crystal was used. The seed was positioned perpendicularly to the planes of the two horizontal plates that restricted growth of $\{11 \overline{1}\}$ facets. With such restriction, the growth proceeded only by $\{100\}$ and $\{102\}$ facets on which the formation of liquid inclusions was rare. The method enabled growth of visibly defect-free crystals of $\sim 10 \mathrm{~cm}$ scale (Figure 2c) at accelerated growth rates up to $6 \mathrm{~mm} /$ day in horizontal direction. Among the three crystals selected for the present studies, two (\# 201 and \#160) were grown using a "point" seeds that allowed growth of all facets at comparable growth rates. The dimensions of the "point" seeds that had vertical sides cut and polished close to the orientations of $\{100\}$ and $\{102\}$ faces were $\sim 5-6 \mathrm{~mm}$ in cross section and about $1 \mathrm{~cm}$ in height. The crystal \#285 was grown on a rod-shaped seed that had similar orientation and cross section, but vertical size of about $5 \mathrm{~cm}$, so that soon after the initial seed regeneration, growth of the $\{111\}$ faces was restricted by the upper bar of the platform.

\section{X-ray diffraction topography and sample preparation}

The X-ray topographic studies were performed with the transmission technique of A.R. Lang [25-27] using a Rigaku camera suitable for crystal slices of diameters up to $10 \mathrm{~cm}$, with conventional X-ray tubes as a radiation source. Due to the particularly low X-ray absorption of organic crystals containing only carbon and hydrogen, $\mathrm{Cu}-\mathrm{K} \alpha$ radiation $(\lambda=1.54 \AA$, linear absorption coefficient of stilbene $\mu \approx 0.5 \mathrm{~mm}^{-1}$ ) is most suitable, allowing the transmission 
sufficient for high intensity and resolution for slices up to $4 \mathrm{~mm}$ in thickness. The topographs were recorded on fine-grained X-ray film Agfa Structurix D4.

Slices of 2-4 mm thickness were cut from the crystals using a solution thread saw with toluene or $m$-xylene as solvents. The slight grooves of the cut surface were removed by gentle polishing on a soft tissue soaked with the same solvents. Despite this treatment slight mechanical surface damages are left. These are removed by rinsing the plate for a few seconds in toluene or xylene, immediately followed by squirting off the still adhering solvent with distilled water in order to prevent further dissolution and subsequent crystallisation. During this procedure inhomogeneous temperature changes, even by a few degrees, should be avoided in order to prevent cracks. The final slice was suspended between mylar foils stretched over circular frames and inserted into the sample holder of the camera. The orientation of the plates cut out of crystals is essential for the presentation of growth defects and their characterization. The best choice is a cut through the seed crystal parallel to the normals of the main growth faces. Since growth dislocations originating from the seed crystal usually propagate more or less perpendicular to the growth faces, they are imaged along their whole length or at least along a considerable part of it [28-31]. In addition, the orientation of the cut should allow the exposure of transmission topographs in at least two different X-ray reflections with large structure factor. The character of the defects (e.g. the Burgers vector of dislocations) can be determined from the variation of the image contrast of the defects in different reflections. Reflections 400, 102, 202, $\overline{2} 02$ and $\overline{1} 11$ have been applied. A review on X-ray diffraction topography with emphasis on organic crystals is given by Klapper [31].

\section{Results}


Fig. 3 is a sketch of crystal \#201 showing the arrangement of growth sectors and the position of cuts P1 and P2 for X-ray topography. Figures 4 and 5 present the topographs of these cuts. Plate P1 (Figure 4) was cut from the bottom side of the crystal just above the flat platform on which the crystal was grown. It contains a part of the seed crystal $\mathrm{S}$ and growth sectors of type $\{100\}$ and $\{102\}$ showing more or less pronounced growth banding. Many growth dislocations originate from the regeneration zone around the seed, partially in bundles of high density beyond X-ray topographic resolution. They exhibit the typical geometry of dislocations in crystals grown under low thermal stress on planar (habit) faces: they are strictly straight-lined with directions depending on the growth direction of the face on which they emerge, and on the Burgers vector [26-29]. Dislocations of bundles A (Figure 4a) are

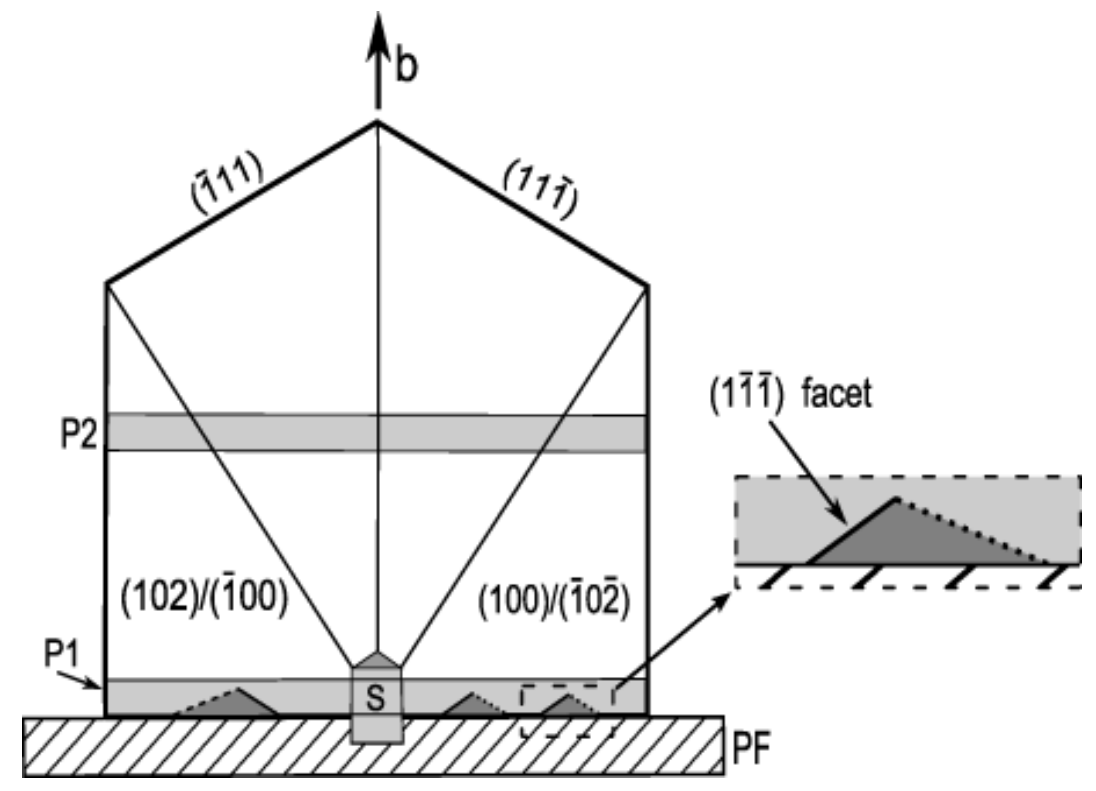

Figure 3: Schematic sketch of crystal \#201, grown on platform PF on a point seed S, with arrangement of growth sectors, illustration of $\{\overline{1} 11\}$ facet formation at the bottom side of the crystal (see enlarged section), and positions of plates P1 and P2 used for X-ray topography.

'extinct' in reflection 400 (Figure 4b, diffraction vector parallel to the dislocation lines), indicating that they have predominantly edge character with Burgers vector either [010] or [001]. The Burgers vectors of the other dislocations could not definitely be determined because of unclear extinction in the topographs of the reflections used for imaging. 
An interesting feature, consisting of a row of triangular contrasts, appears along the long diagonal of the plate (i.e. the boundary between $\{102\}$ and $\{100\}$ growth sectors). It

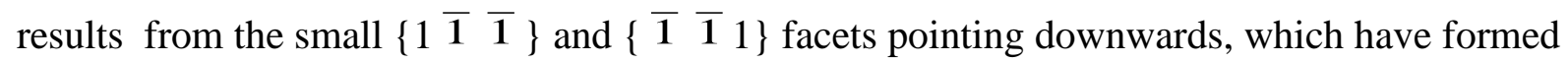
in a quasi-periodic manner and grown out again, thus forming a series of small growth sectors of type $\{11 \overline{\mathbf{1}}\}$, as illustrated in Figure 3. The X-ray topographic contrast results rather from the boundaries of these sectors than from their bulk. In some cases small inclusions have formed on the facets, which are the origin of growth dislocations (see enlarged section in Figure 4c). These dislocations, 'extinct' in reflection 400, are predominantly screw (Burgers vector [001]) and have grown out together with the facet growth sectors. Figure 5a shows the topograph of the plate $\mathrm{P} 2$, which was cut from the original crystal about $3 \mathrm{~cm}$ above $\mathrm{P} 1$ ( $c f$. Figure 3). The arrangement of growth sectors is outlined in Figure 5b. Whereas the boundaries between sectors $\{102\}$ and $\{100\}$ are undisturbed (no contrast), those between $\{102\} \&\{\overline{\mathbf{1}} 11\}$ and $\{100\} \&\{\overline{\mathbf{1}} 11\}$ appear, due to their inclination toward the plate surface, as broad contrast bands, indicating a considerable degree of lattice distortion. The dislocations in sectors $\{\overline{\mathbf{1}} 11\}$ penetrate the plate more or less steeply, depending on their Burgers vectors. They originate from the regeneration zone at the seed and from liquid inclusions in the crystal region underneath $\mathrm{P} 2$. Some of them penetrate the boundary into a $\{102\}$ or a $\{100\}$ sector and are bent in a sharply defined direction parallel to the (010) plane (i.e. parallel to the plate surface). 


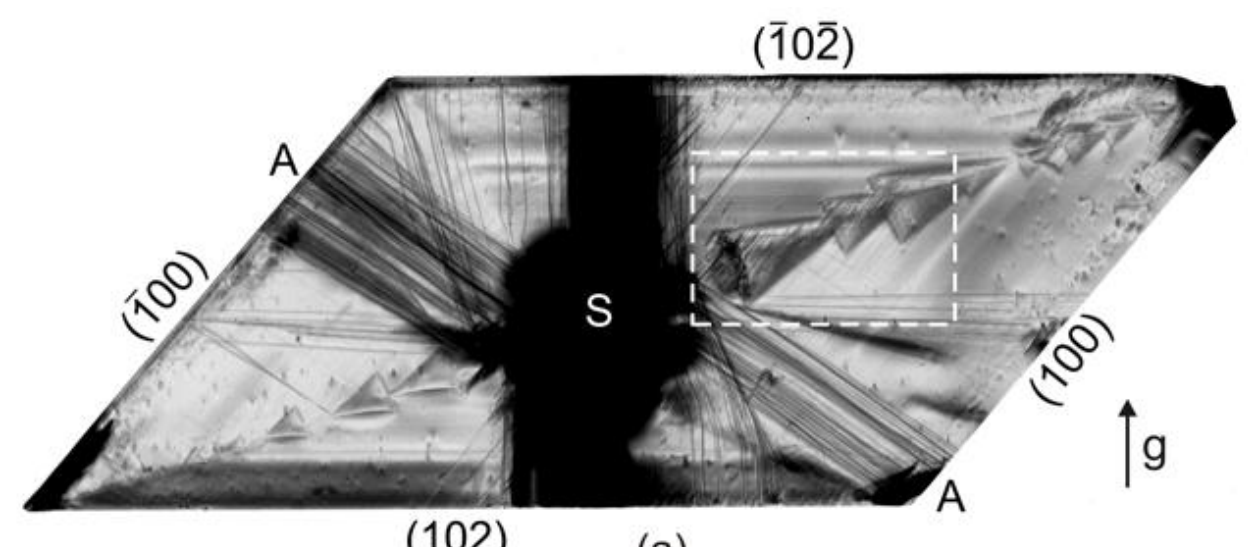

(102)

(a)

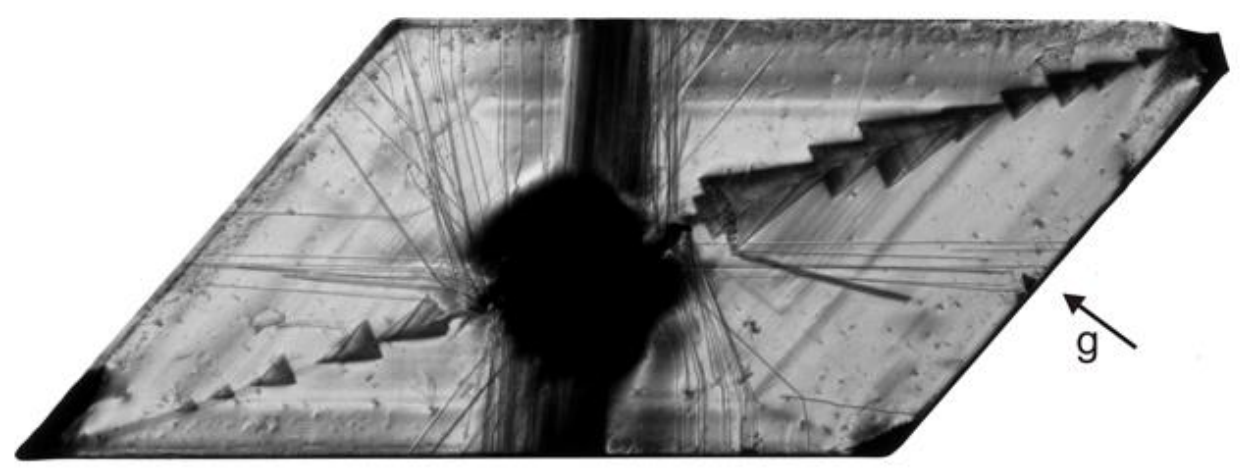

(b)

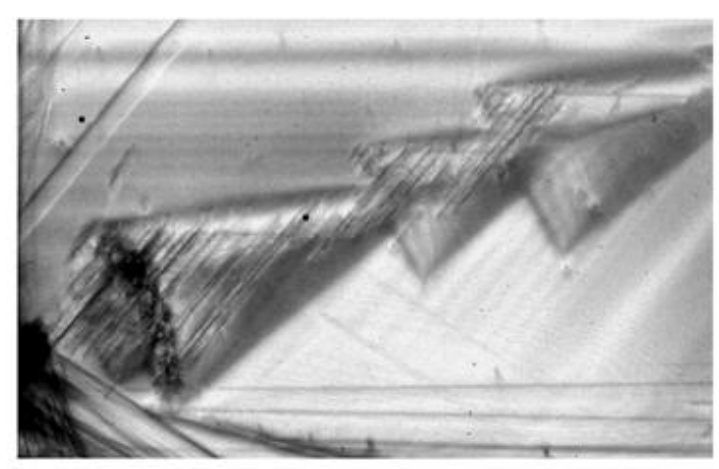

(c)

Figure 4: Plate P1 of crystal \#201, cut through the seed crystal S, $5.5 \times 2 \mathrm{~cm}^{2}$, about $3.5 \mathrm{~mm}$ thick. (a) Topograph in reflection 102. (b) Reflection 400. Arrows g: diffraction vectors. Triangular contrast features along long diagonal of the plate originate from $\left\{\begin{array}{ll}\overline{1} & 11\end{array}\right\}$ facet sectors which have formed at the bottom side of the crystal (cf. Fig. 3). A: Bundles of edge dislocations 'extinct' in topograph (b). The small dot contrasts are due to small surface damages. (c) Enlarged section from topograph (a), showing screw dislocations in the $\begin{cases}\overline{1} & 11\}\end{cases}$ facet sectors. 


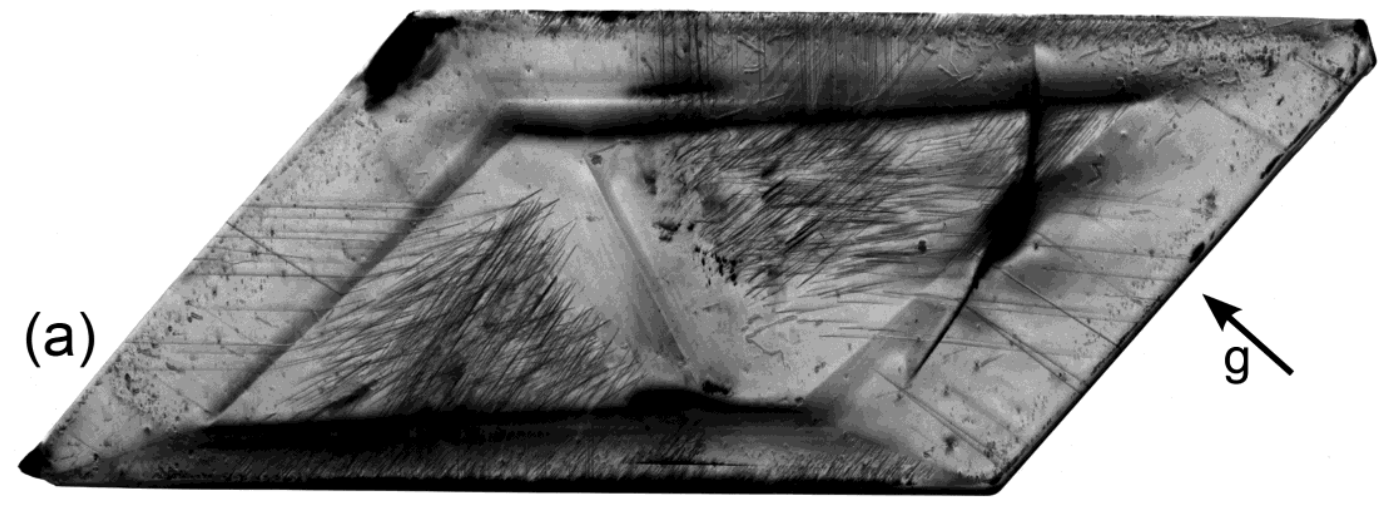

(b)

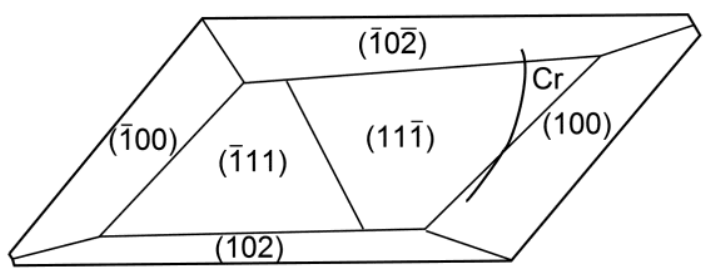

Figure 5: Plate P2 of crystal \#201, cut about $3 \mathrm{~cm}$ above P1, same size and thickness as P1. (a) Topograph reflection 400; $\mathbf{g}$ diffraction vector. (b) Sketch of growth sectors. Cr: crack.
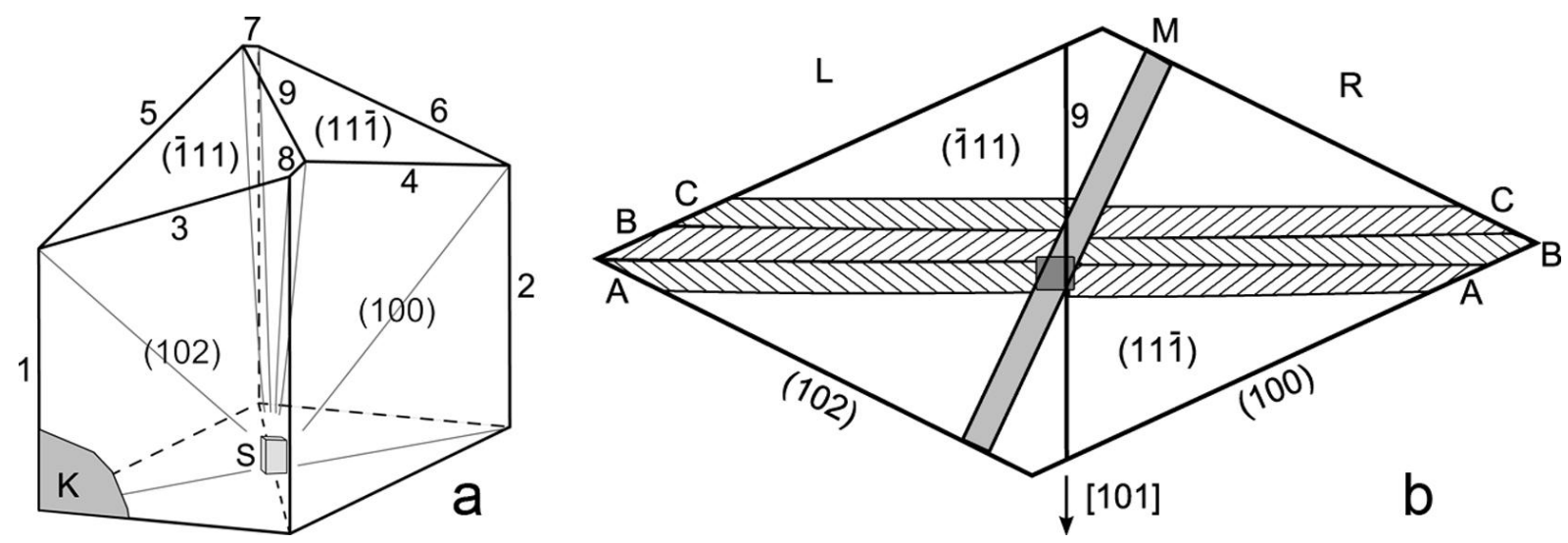

Figure 6: (a) Sketch (inclined view) of crystal \#160, grown on a dome-shaped platform (not shown here) with the point seed $S$ fixed in flat pit in the cusp. Size: ca. $60 \mathrm{~mm}$ wide, $56 \mathrm{~mm}$ high. The numbers mark the crystal edges which define the growth-sector boundaries in the interior of the grown crystal. The thin lines originating from the seed $\mathrm{S}$ depict the path of the crystal corners (constant growth velocities of all faces provided). They represent the edges of the growth sectors, which have the shape of an inclined pyramid with its apex pointing to the seed and the final growth face as basis. At the bottom left corner $\mathrm{K}$ an unwanted secondary crystal had appeared and grown with the main crystal. It broke off after growth. (b) Top view of the crystal with approximate plan of crystal cuts for topography. First, plate M was cut through the seed crystal, then plates A, B, C were prepared from the remaining left and right halves L and R. The latter are perpendicular to the [101] edge (9) of the crystal. Note that by cutting and polishing a crystal layer of about $0.5 \mathrm{~mm}$ is lost. 
For a representative survey of the typical growth defects in trans-stilbene, a selected crystal (\#160) was cut into several slices of optimal orientation. The shape of the crystal and the cutting plan of the slices are shown in Figure 6a and b. These cuts allow a comprehensive recording of inclusions, growth sectors and their boundaries, striations and dislocations in reflection $20 \overline{2}$ with a suitable structure factors. The topographs of these slices, as well as enlarged sections out of them, are shown in Figures 7-9 and 11, 12. The essential details visible in these topographs are described and explained in the corresponding figure captions.

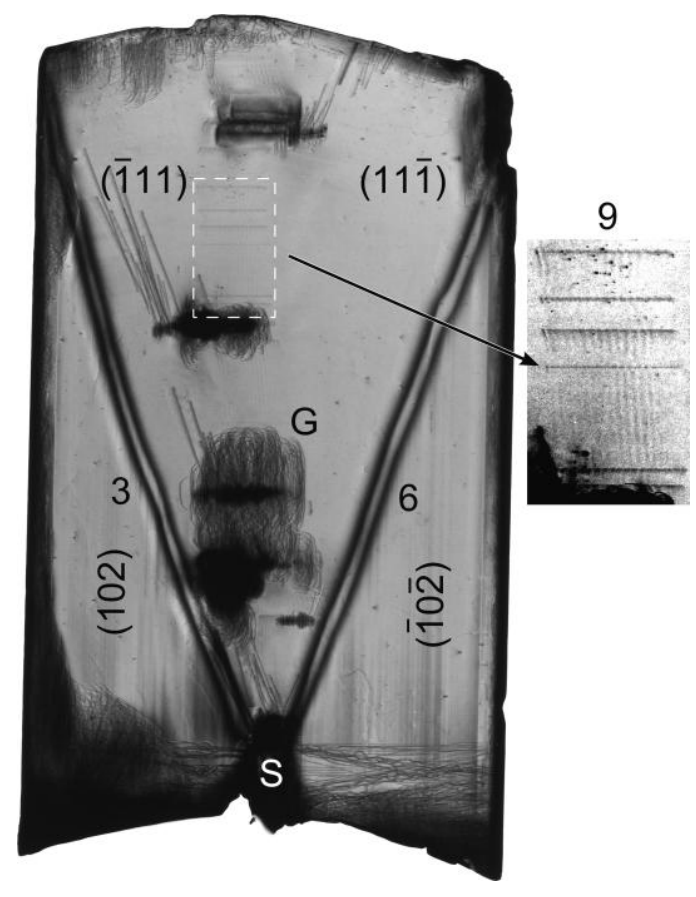

Figure 7: X-ray topograph (reflection 202) of the plate M of Figure 6b, $56 \mathrm{~mm}$ high, about 4 $\mathrm{mm}$ thick. Monoclinic axis [010] vertical. The concave shape of bottom edge with the seed crystal is due to the dome-shaped platform. The double bands (3) and (6) with strong kinematical contrast are sector boundaries between $\{102\}$ and $\left\{\overline{1}_{1} 11\right\}$ growth faces. They are associated to the edges (3) and (6) in Figure 6a. The two lines of high contrast originate from the intersections of the boundaries with the plate surface ( $c f$. Discussion). The boundary between the neighboured $\{\overline{\mathbf{1}} 11\} /\{11 \overline{\mathbf{1}}\}$ sectors (associated with edge 9, i.e.[101], cf. Figure 6 ) penetrates the plate under an angle of $26^{\circ}$ and appears as very faint dynamical fringe contrast, which is shown by contrast enhancement in the enlarged section. The horizontal linear marks are correlated with growth fluctuations. Numerous glide dislocation half-loops originate from the liquid inclusions (e.g. G) in the $\{\overline{\mathbf{1}} 11\} /\{11 \overline{1}\}$ sectors. They arise by relaxation of stress associated with the inclusions, which are also the origin of growth dislocations. The latter, however, have directions steeply inclined with respect to the plate surface ( $c f$. Figure 8, topographs of plates $\mathrm{R}$ ) and thus emerge from the plate more or less close to the inclusion. 


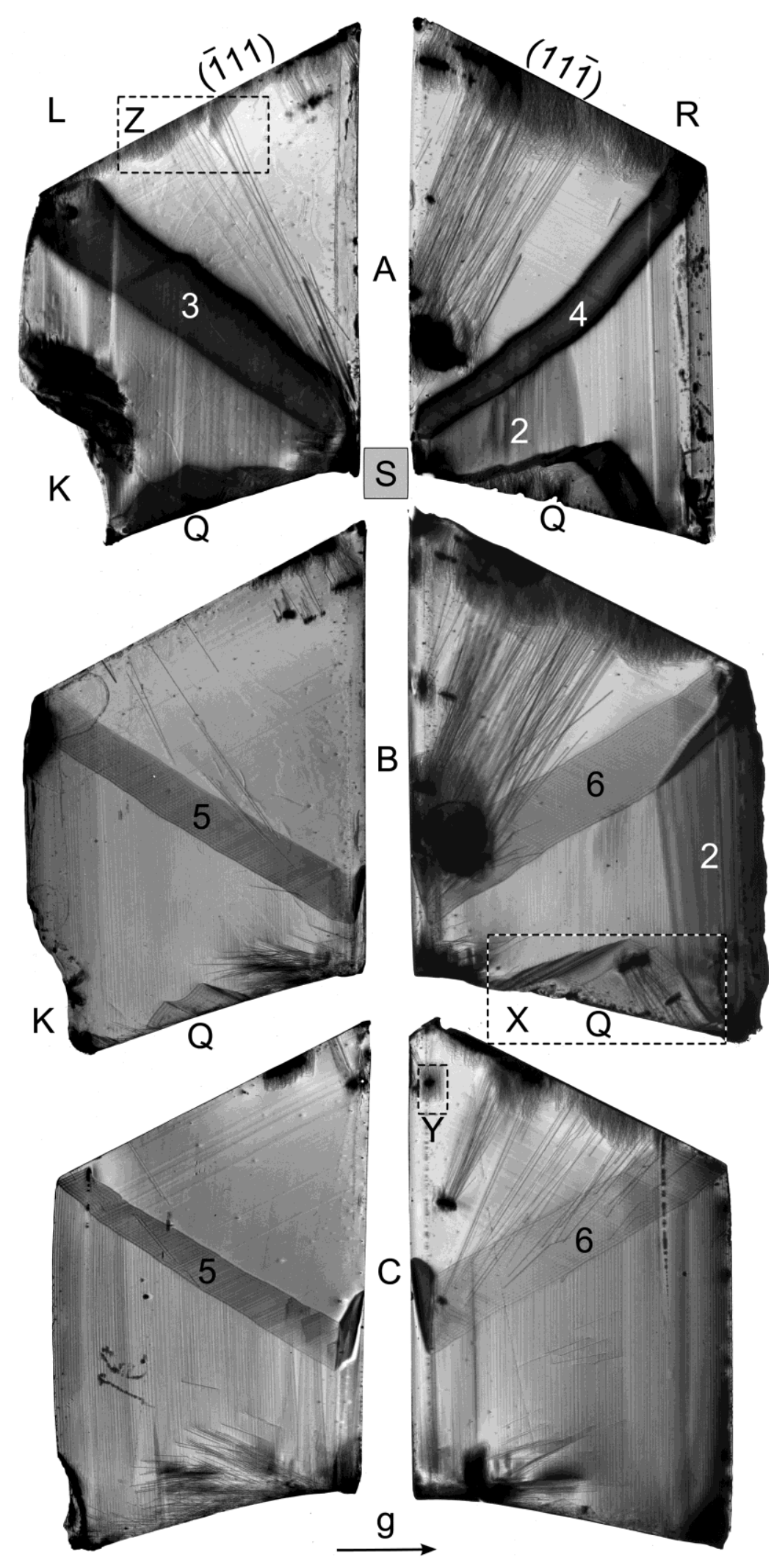


Figure 8: Topographs of the six plates A, B, C (left L and right $\mathrm{R}$ ) shown in the cutting plan of crystal \#160 in Figure 6b, all $56 \mathrm{~mm}$ high, between 2.5 and $4.0 \mathrm{~mm}$ thick, reflection $20 \overline{2}$. S: position of the seed crystal. The numbers (1), (2), ....etc. denote the growth-sector boundaries associated to the crystal edges shown in Figure 6a. The boundaries (3), (4) [in plates A] and (5), (6) [in plates B and C] intersect the plates and appear by broad contrast bands, their widths depending on the intersection angle and the plate thickness. The wide contrast (2) in the topographs of slices A (R) and B (R) arises from the (100)/( $\overline{1} 0 \overline{2})$ sector boundary [edge (2) in Figure 6a], which is nearly parallel to the plate surface. A large part of this boundary is eliminated by the cut between plates A and B, as is the total corresponding boundary (1) in the left part L. The contrast features Q result from the growth sectors of $\{\overline{\mathbf{1}} 11\}$ and $\{11 \overline{\mathbf{1}}\}$ facets, temporarily formed and grown out again, analogous to those shown in Figures 3 and 4 (see enlarged section $X$ in Figure 9). The liquid inclusions in sectors $\{\overline{\mathbf{1}} 11\}$ and $\{11 \overline{\mathbf{1}}\}$ are the origin of growth dislocations as well as of glide dislocations. Numerous glide dislocations have also entered after growth through the ( $\overline{\mathbf{1}} 11)$ and $(11 \overline{\mathbf{1}})$ surfaces (see enlarged section $\mathrm{Z}$ in Figure 12).

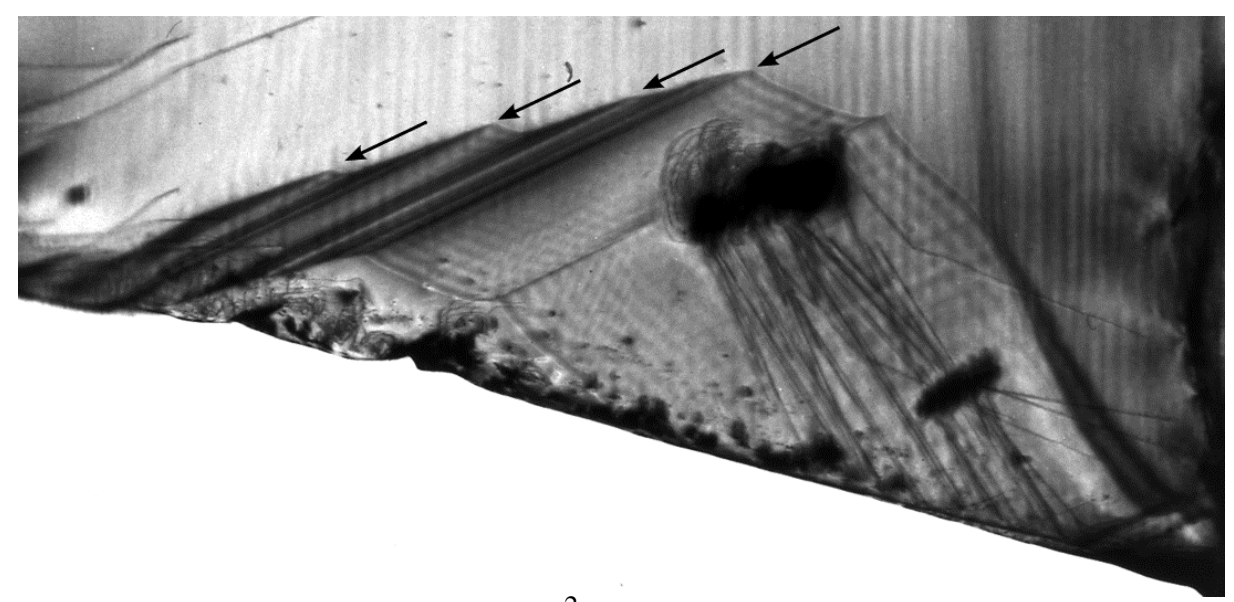

Figure 9: Enlarged section $X\left(\approx 27 \times 13 \mathrm{~mm}^{2}\right)$ of topograph $B(R)$ of Figure 8, showing a $\{1 \overline{\mathbf{1}} \overline{\mathbf{1}}\}$ facet sector. Due to local growth fluctuations the facet appears by strong kinematical contrast (arrows) indicating considerable lattice strain. Later a group of small liquid inclusions has been formed. It is the origin of growth dislocations, which grew out with the sector, and of glide dislocations. The boundary to the neighboured ( $\overline{1} 0 \overline{2}$ ) sector is inclined to the plate surface by a small angle and appears by wide fringe contrast. 

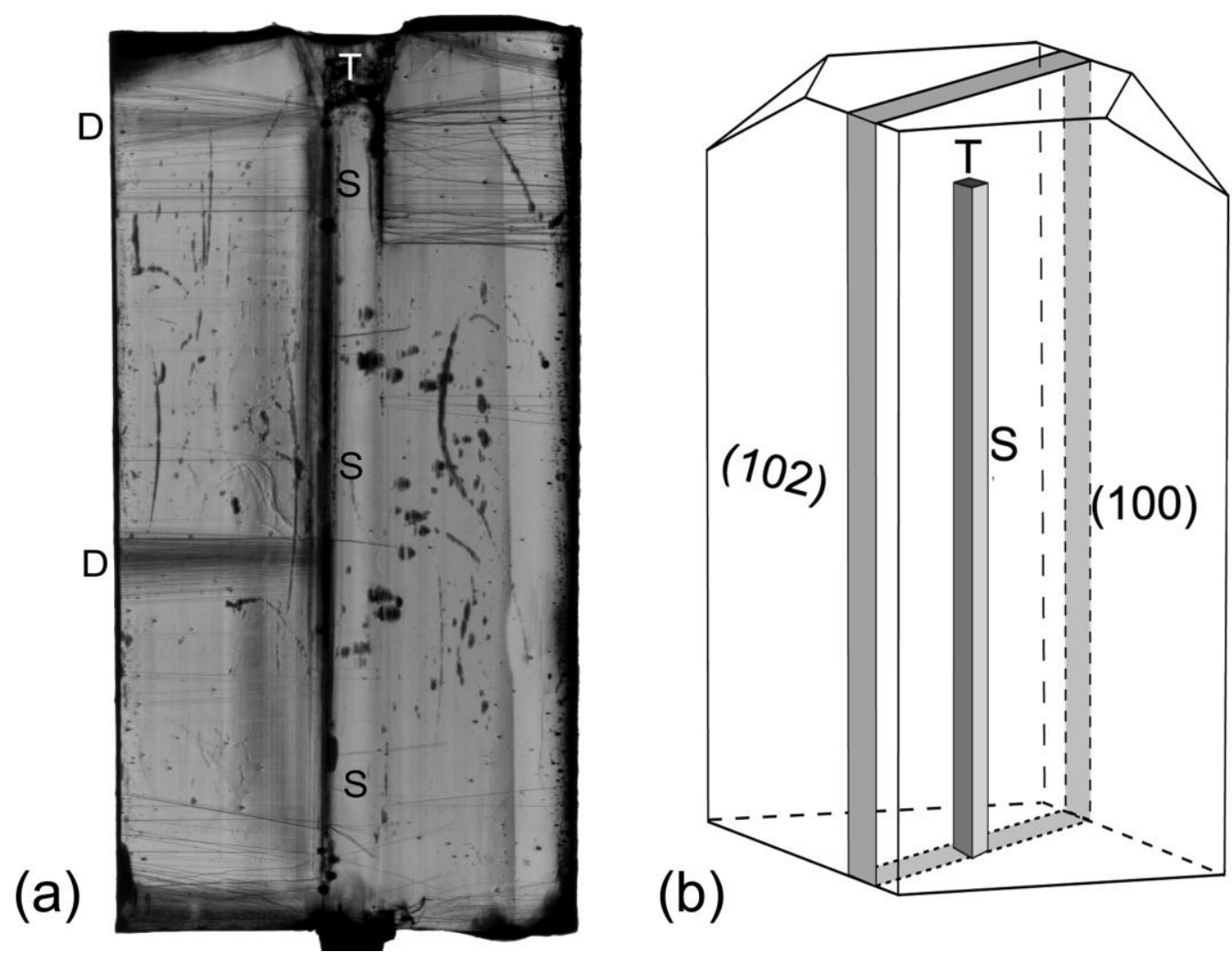

Fig. 10: (a) Topograph of a slice (55 mm high, $3.4 \mathrm{~mm}$ thick, reflection 102), cut from crystal \#285 through the seed rod along its full length, as shown in (b). The growth of this crystal was restricted in the vertical direction (monoclinic axis), so that the major part has grown on 1102) and (100) faces. Due to the gap between the top end of the seed and the confining bar small sectors of $\{11 \overline{\mathbf{1}}\}$ facets (not contained in this cut) have formed. The dislocations originating from the seed regeneration zone are typical and representative also for "fully restricted" crystals grown without the gap T (e.g. Fig. 2c). They are often arranged in bundles $\mathrm{D}$ which correlate with some of the growth hills visible on the face during growth. The irregular black spots are due to mechanical damages of the plate surface.

Figure 10a presents the topograph of a plate cut from crystal \#285 as shown in Figure 10b. It was grown on a $50 \mathrm{~mm}$ long [010] seed rod with vertical restriction of growth. Thus the main body of the crystal has grown on $\{102\}$ and $\{100\}$ faces and is free from liquid inclusions except some small ones in the seed regeneration zone. As usual, growth dislocations originate from defective spots of the regeneration zone around the seed rod and propagate mainly normal to the $\{102\}$ growth faces. Some of them form bundles D which are associated with pronounced growth hills on the growth face sometime clearly visible in reflected light. Due to the gap (about $5 \mathrm{~mm}$ ) between the top end of the seed rod and the restricting barrier small 
$\{11 \overline{\mathbf{1}}\}$ facets with regeneration zone $\mathrm{T}$ have formed. These can be completely eliminated by avoiding the gap $\mathrm{T}$, as was done for the crystal shown in Figure 2c.

\section{Discussion}

\subsection{Inclusions}

In our crystals of trans-stilbene, grown under the conditions described in Section 3, liquid inclusions have been very frequently formed in the $\{\overline{\mathbf{1}} 11\}$ growth sectors. On the other hand, the $\{100\}$ and $\{102\}$ sectors in most cases grew free of inclusions. This may be due to the molecular structure of the growth surface, which is more rugged for faces favouring liquid inclusions. In the present case, however, there is a strong indication that a kinetic reason plays an important role: macro-steps formed at the edges between $\{100\}$ or $\{102\}$ and $\{11 \overline{\mathbf{1}}\}$ faces (e.g. edges 3, 4, 5, 6, and 9 in Figure 6a) propagate toward the centre of the

$\{\overline{1} 11\}$ faces and form a depression which is finally overgrown by growth layers, thus capturing solution. A similar effect has been observed and described by Zaitseva et al. [32] for KDP. The mechanism of such inclusion formation in trans-stilbene and other organic crystals is the subject of current and future studies, the results of which will be presented in a separate publication.

\subsection{Striations and growth-sector boundaries}

Striations and faulty growth-sector boundaries are common in crystals grown from melts and solutions containing impurities. In crystals grown from solutions constituents of the 
solvent (e.g. molecules) may contribute to these imperfections". Whereas striations are associated with fluctuations of growth conditions and thus are arranged as strata parallel to the growth faces, growth-sector boundaries are formed by the path of the edges between neighboured faces during growth. Growth sectors of the planar faces have the shape of a pyramid (in general inclined), with their apices pointing towards the seed and the final growth faces as basis (cf. Figure 6a).

The subdivision of our stilbene crystals in their various growth sectors is shown in Figures 4, 5 and 6a. By X-ray topography both striations and sector boundaries are usually depicted by kinematical contrast due to lattice distortions resulting from inhomogeneities (striations) or accumulation of impurities (boundaries). Lattice distortions along sector boundaries may also arise from slightly different $d$-values of lattice planes parallel to the growth faces. Examples of boundaries with strong kinematical contrast are those labelled 3 and 6 in Figure 7, and 3 and 4 in Figures 8 A(L) and 8 A(R). They show strongly increased contrast along their lines of outcrop from the plate surface. These boundaries are accompanied by stress, which relaxes at the plate surfaces during cutting, thus forming two lines of increased strain which produces increased kinematical contrast. This is strikingly shown in Figure 7 (boundaries 3 and 6). The boundaries 5 and 6 in the plate pairs B and C of Figure 8 appear by dynamical fringe contrast. This contrast arises either from a displacement of the lattices of the adjoining regions (similar to the fault vector of a stacking fault) or a very small lattice tilt of the order of seconds of arc. A detailed characterisation of these boundaries by comparing several topographs in different reflections and by 'section topography' [26, 33], however, is beyond the scope of this study. Finally it is mentioned that kinematical or dynamical topographic contrast is usually observed only for boundaries between

\footnotetext{
In the present case of stilbene there are indications that the striations are mainly caused by step generation from the crystal edges [32].
} 
symmetrically non-equivalent sectors, whereas boundaries between equivalent sectors are mostly free of contrast, indicating the absence of lattice disturbance. This is also found in the present study: boundaries 1-6 (Figure 6a) between non-equivalent sectors are clearly depicted (Figures 7 and 8), whereas the boundary 9 between equivalent sectors ( $\overline{\mathbf{1}} 11)$ and $(11 \overline{\mathbf{1}})$ is hardly visible (Figure 7, enlarged section).

\subsection{Dislocations}

With respect to their origin, two categories of dislocations are distinguished:

(1) Growth dislocations, connected to and propagating with the growth face. They exhibit the typical geometry observed in crystals grown under stress-free conditions on planar surfaces $[28,29]$. They are straight-lined with directions depending on their Burgers vector and the growth face to which they are connected. When crossing over an edge to a neighboured growth face, they are bent into a new direction typical for the new growth direction. A comprehensive treatment of these preferred directions of minimum energy is presented in [28, 29].

(2) Glide dislocations, formed 'behind' the growth front by plastic processes driven by the relaxation of stress induced around inclusions during the cooling period of the crystal at the end of the growth run ('post-growth dislocations'). They usually form loops or half-loops emitted from inclusions (e.g. label G in Figure 7, and Figure 11).

Both kinds of dislocation have been observed in the present study of trans-stilbene crystals and are described in the preceding chapters. A comprehensive review of growth and postgrowth dislocations and their interactions is presented in [28]. 


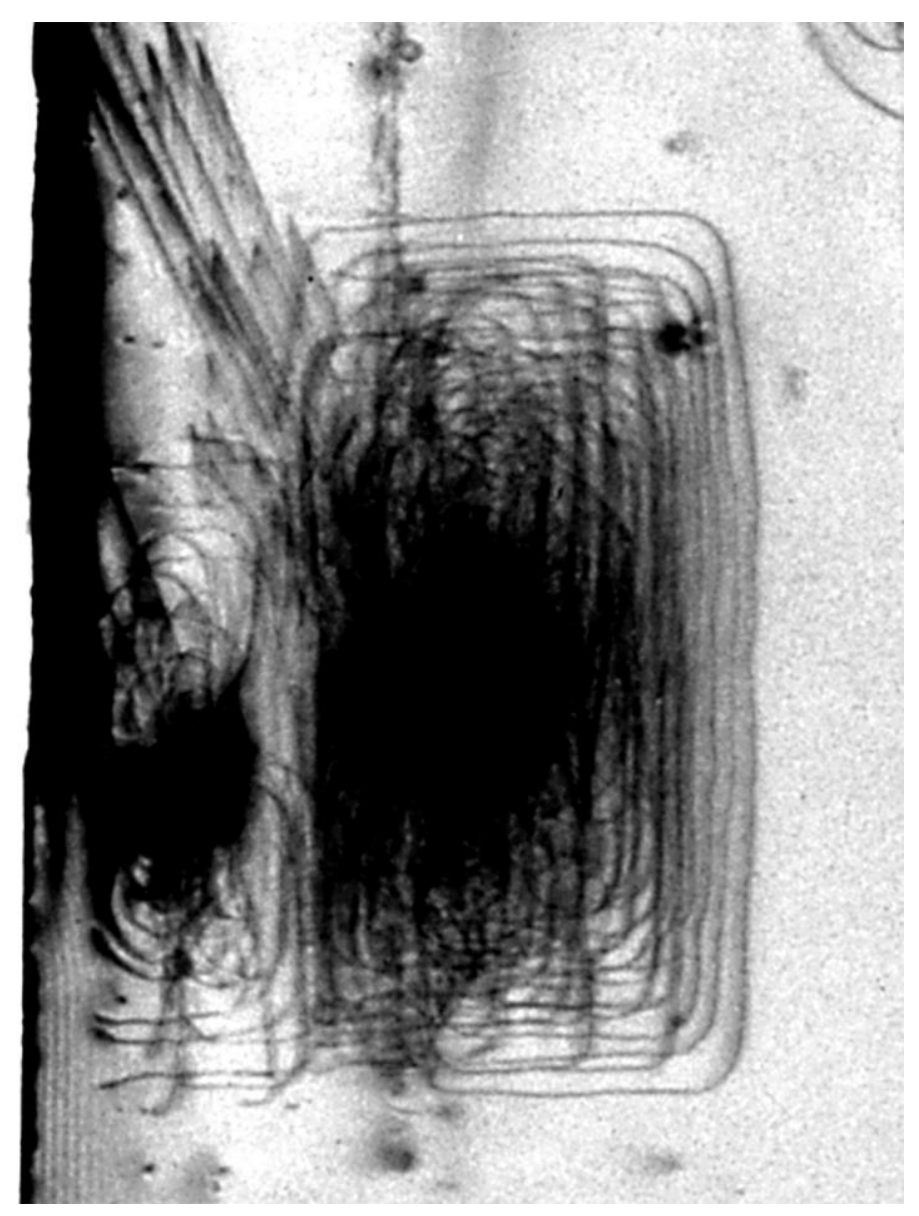

Figure 11: Enlarged section $Y\left(\approx 4 \times 5 \mathrm{~mm}^{2}\right)$ of the topograph $C(R)$ of Figure 8, showing glide dislocations in the form of closed loops emitted from liquid inclusions by stress relaxation. The arrow-like lines in the upper left corner are growth dislocations originating from the large inclusion and emerging from the X-ray exit surface.

In the study of dislocations by X-ray topography, the Burgers vectors of dislocations are often determined by the variation of the dislocation contrast in different imaging reflections. In such variation, the contrast is maximal for reflecting planes perpendicular to the Burgers vector, and minimal or zero ('extinct') for planes parallel to the Burgers vector $(\mathbf{g} \cdot \mathbf{b}$ criterion [26, 27]; g: diffraction vector, b: Burgers vector). In the present case, however, this did not definitely work (except for the pure-edge dislocation bundle A in Figure 4), obviously due to the elastic anisotropy with various elastic cross-coupling constants of the monoclinic crystal. 
The glide dislocations deserve a particular discussion. The plastic deformation of crystals is usually described in terms of glide systems $[u v w](h k l)$, where $(h k l)$ is the glide plane

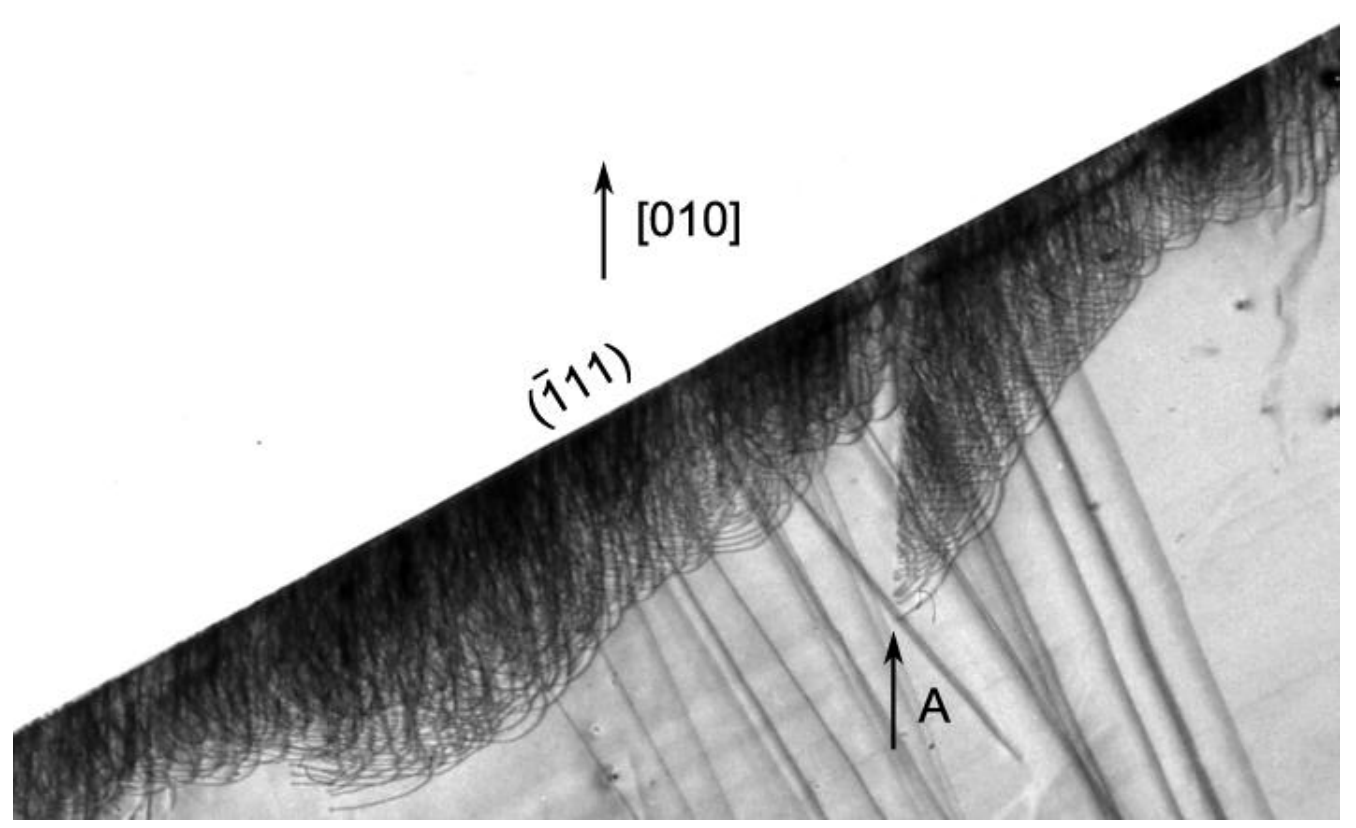

Figure 12: Enlarged section $\mathrm{Z}\left(\approx 16 \times 10 \mathrm{~mm}^{2}\right)$ of the topograph $\mathrm{A}(\mathrm{L})$ of Figure 8 , showing numerous glide dislocations which have entered the crystal after growth through the ( $\left.\begin{array}{l}\overline{1} \\ 11\end{array}\right)$ surface by mechanical impacts during handling of the crystal. The arrow indicates the emergence from the plate surface of a group of dislocations located in a defined (glide) plane.

(usually one of the three lattice planes with largest interplanar spacing) and $[u v w]$ the glide direction (i.e. the Burgers vector, usually the shortest lattice translation within the glide plane). The three shortest lattice translations of trans-stilbene are the three basis vectors $\mathbf{b}=$ [010] with $5.723 \AA$ Á, $\mathbf{c}=[001]$ with $12.381 \AA$ and $\mathbf{a}=$ [100] with $15.710 \AA \AA$. The three lattice planes with largest $d$-values are (100) with $d=14.58 \AA \AA,(001)$ with $d=11.55 \AA$ and ( $\overline{1} 01)$ with $d=11.31$ Á. Since the Burgers vector could not be determined from the visibility rules (g. b criterion) of the dislocation images in different reflections, other indicative features have to be applied. These are: 
The width of the X-ray topographic image of the glide dislocations is considerably smaller than that of the other dislocations ( $c f$. Figure 11), indicating a substantially shorter Burgers vector.

(ii) The half-loops of the glide dislocations emitted from inclusions are clearly elongated in direction of the [010] (monoclinic axis), indicating this as the direction of easiest glide ( $c f$. label G in Figure 7).

(iii) Glide dislocations introduced into the crystal through the surface due to mechanical impacts during handling enter the crystal practically only through the faces ( $\overline{\mathbf{1}} 11)$ and $(11 \overline{\mathbf{1}})$, which have a normal component along the monoclinic axis [010] ( $c f$. Figure 11). Only very few glide dislocations enter through faces (100) and (102), which are parallel to the direction [010] of easiest glide, although these faces are also subject to similar mechanical impacts during handling as the former ones. If such dislocations occur, they are associated with spots of heavy mechanical surface damages.

These features provide strong evidence that $\mathbf{b}=[010]$ with the shortest lattice translation is the Burgers vector of the glide dislocations. Information on the glide plane can be obtained from the feature indicated in Figure 12 by the arrow, which shows the emergence points of a group of glide dislocations from the plate surface. Since these points lie quite accurately on a line parallel to the monoclinic direction [010], the dislocations of this group must be situated in a plane belonging to the zone axis [010]. Because there is no such line belonging to the emergence from the other opposite face of the plate (thickness about $4 \mathrm{~mm}$ ), the inclination $\alpha$ of this plane must be quite small*. The following low-indexed lattice planes are considered:

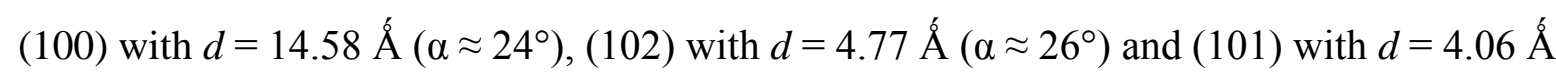
$\left(\alpha \approx 18^{\circ}\right)$. Among these the $d$-value of plane (100) is by far the largest, and we can conclude 
that the observed glide dislocations belong to the glide system [010](100) with the largest interplanar spacing of the glide plane (100) and the shortest translation [010] (Burgers vector) of the trans-stilbene lattice as glide direction.

\footnotetext{
*An accurate determination of the inclination angle from various projections using different $\mathrm{x}$-ray reflections is very elaborate and was not done.
}

\section{Conclusion}

The present study has shown that the overall perfection of a molecular crystal (trans-stilbene) grown from organic solution is comparable to that of inorganic crystals grown from aqueous solutions. The results of $\mathrm{x}$-ray topographic studies indicate that the general nature of defects is in accordance with the defects observed previously in rapidly grown KDP crystals [22-24]. Growth defects such as inclusions, dislocations, growth striations, growth bands, and faulted growth-sector boundaries occur in the same typical arrangements and geometries as is observed in all kinds of crystals growing on habit faces from solution [28, 29]. However, the frequency of liquid inclusions (and of dislocations associated to them) has been found considerably enhanced in trans-stilbene crystals. Since the investigated crystals were grown at relatively slow growth rates, not faster than $\sim 3 \mathrm{~mm}$ /day for individual faces, in comparison to $10-20 \mathrm{~mm} /$ day used in rapid growth of KDP, the origin of these inclusions can hardly be attributed to the traditional explanation of higher inhomogeneities of supersaturation across the growth face. It seems more likely that in trans-stilbene growth of certain faces may be dominated by a mechanism that involves step generation on the crystal edges and corners. This mechanism observed in addition to the dislocation growth in rapidly grown KDP crystals [32] will be presented in future publications as a part of studies whose aim is the development 
of rapid growth techniques for production of trans-stilbene and other organic crystals for technical and scientific applications.

\section{Acknowledgement}

The authors wish to thank Mrs. A. von Berg and Mr. Jan M. Simons (Aachen) for their help in the preparation of figures. The crystal growth work was performed under the auspices of the US Department of Energy by Lawrence Livermore National Laboratory under ContractDEAC5 07NA27344.

\section{References}

1. J. B. Birks, The theory and practice of scintillation counting, Pergamon Press, London 1954.

2. F. D. Brooks, A scintillation counter with neutron and gamma-ray discriminators, $\mathrm{Nucl}$. Instrum. Methods 4 (1959) 151-163.

3. F. D. Brooks, Development or organic scintillators. Nucl. Instrum. Methods 162 (1979) 477-505

4. G. T. Wright, Scintillation decay times of organic crystals. Proc. Phys. Soc. B69 (1956) 358-372. 
5. R. B. Owen, The decay times of organic scintillators and their application to the discrimination between particles of different specific ionization. I.R.E. Trans. Nucl. Sci. NS-5 (1958) 198-201.

6. L. M. Bollinger and G. E. Thomas. Measurement of the time dependence of scintillation intensity by a delayed coincidence method, Rev. Sci. Instrum. 32 (1961) 1044-1050.

7. Amcrys-H, http://www.amcrys-h.com

8. N. Z. Galunov, O. A. Tarasenko, V. A. Tarasov. Determination of the light yield of organic scintillators. Functional Materials 20 (2013) 304-309.

9. L. Carman, N. Zaitseva, H. P. Martinez, B. Rupert, I. Pawelczak, A. Glenn, H. Mulcahy, R. Leif, K. Lewis, S. Payne. The effect of material purity on the optical and scintillation properties of solution-grown trans-stilbene crystals. J. Cryst. Growth 368 (2013) 56-61.

10. N. Zaitseva, A. Glenn, L. Carman, H. P. Martinez, R. Hatarik, H. Klapper, S. Payne. Scintillation properties of solution-grown trans-stilbene crystals. Nucl. Instrum. Methods 789 (2015) 8-15.

11. Inrad Optics, http://www.inradoptics.com

12. N. P. Zaitseva, L. Carman, A. Glen, J. Newby, M. Faust, N. Cherepy, S. Payne. Application of solution technique for rapid growth of organic crystals. J. Crystal Growth 314 (2011) 163-170.

13. G. Hull, N. Zaitseva, N. Cherepy, J. Newby, W. Stoeffl, and S. Payne, New organic crystals for pulse shape discrimination, IEEE Transactions on Nuclear Science 56 (2009) 899-903.

14. R. Hatarik, L. A. Bernstein, J. A. Caggiano, M. L. Carman, D. H. G. Schneider, N. P. Zaitseva, and M. Wiedeking, Characterizing time decay of bibenzyl scintillator using time correlated single photon counting, Rev. Sci. Instr. 83 (2012) 10 D911. 
15. P. Groth, Chemische Krystallographie, Vol. 5, p. 192-193. Wilhelm Engelmann, Leipzig 1919. (Photo-reproduction by The Groth Institute, University Park, Pennsylvania, USA, 1959).

16. J. M. Robertson, W. Woodward, X-ray analysis of the dibenzyl series. IV. Detailed structure of stilbene. Proc. Roy. Soc. (London) A162 (1937) 568-583.

17. C. J. Finder, M. G. Newton, N. L. Allinger, An improved structure of trans-Stilbene. Acta Cryst. B30 (1974) 411-415.

18. J. Bernstein, Refinement of trans-Stilbene: a comparison of two crystallographic studies. Acta Cryst. B31 (1975) 1268-1271.

19. A. Hoekstra, P. Meertens, A. Vos, Refinement of the crystal structure of trans-stilbene (TSB). The molecular structure in the crystalline and gaseous phases. Acta Cryst. B31 (1975) 2813-2817.

20. S. K. Hutchinson, R. Lapage, Studies of organic phosphors for use as scintillation counters. II. Growth from solution and thermal expansion of stilbene crystals. Atomic Weapons Research Establ., Rep. No. 0.5/53, 1953.

21. S. Haussühl, Elastic and thermoelastic properties of selected organic crystals. $Z$. Kristallogr. 216 (2001) 339-353.

22. N. P. Zaitseva, I. Smolsky, L. N. Raskovich, Study of rapid growth of KDP crystals by temperature lowering. Sov. Phys. Crystallogr. 36 (1991) 113-115.

23. N. P. Zaitseva, L. Carman, Rapid growth of KDP and DKDP crystals, Prog. Cryst. Growth Charact. Mater. 43 (2001) 1-118.

24. N. P. Zaitseva, J. J. De Yoreo, M. R. Dehaven, R. L. Vital, K. E. Montgomery, M. Richardson, Rapid growth of large-scale (40-55cm) $\mathrm{KH}_{2} \mathrm{PO}_{4}$ crystals, J. Crystal Growth 180 (1997) 255-262. 
25. A. R. Lang, Studies of individual dislocations in crystals by X-ray diffraction microradiography. J. Appl. Phys. 30 (1959) 1748-1755.

26. A. R. Lang, Topography, in: International Tables for Crystallography, Vol. C, Ch. 2.7, pp. 113-123. International Union of Crystallography, Kluwer Academic Publishers, Dordrecht 1992.

27. B. K. Tanner, X-ray diffraction topography. Pergamon Press, Oxford 1976.

28. H. Klapper, Generation and propagation of dislocations during crystal growth. Mat. Chem. Phys. 66 (2000) 101-109.

29. H. Klapper, Generation and propagation of defects during crystal growth in: Springer Handbook of Crystal Growth, eds.: G. Dhanaraj, K. Byrappa, V. Prasad and M. Dudley (Springer, Berlin-Heidelberg, 2010) p.93.

30. H. Klapper, P. Rudolph, Defect Generation and Interaction during Crystal Growth, in: Nishinaga, T., Rudolph, P., Kuech, T., (Eds.) Handbook of Crystal Growth, Second Edition, Vol. II b (pp. 1093 -1141 ). Elsevier BV, Netherlands, 2015.

31. H. Klapper, X-ray topography of organic crystals. In: Crystals: Growth, Properties and Characterization, Vol. 13, ed. N. Karl, (Springer, Berlin-Heidelberg 1991) p.109-162..

32. N. Zaitseva; I. Smolski, L. Carman, Growth phenomena in the surface layer and step generation from the crystal edges. J. Crystal Growth 222 (2001) 249-262.

33. A. Authier, Section topography. In: X-ray Optics. Application to Solids. Editor H.-J. Queisser, Ch.5, pp145-189. Springer, Berlin 1977. 